\title{
Changes in Excitability of the Motor Cortex Associated with Internal Model Formation during Intrinsic Visuomotor Learning in the Upper Arm
}

\author{
Timothy Hunter ${ }^{1}$, Paul Sacco ${ }^{2}$, Duncan L. Turner ${ }^{1,3,4}$ \\ ${ }^{1}$ School of Health and Bioscience, University of East London, London, UK \\ ${ }^{2}$ The Pain Research Institute, Division of Neurosciences, University of Liverpool, \\ Aintree Hospital, Liverpool, UK \\ ${ }^{3}$ Department of Clinical Neurosciences, University of Cambridge, Cambridge, UK \\ ${ }^{4}$ Lewin Stroke Unit, Addenbrookes Hospital, Cambridge, UK \\ E-mail: t.hunter@uel.ac.uk \\ Received May 4, 2011; revised June 22, 2011; accepted July 18, 2011
}

\begin{abstract}
Previous studies have shown that the primary motor cortex (M1) may drive part of the feed forward control of well learnt simple movements by specifying patterns of muscle activation. This study explored the role of the M1 in the feed forward control of newly formed movement patterns after motor adaptation. Ten healthy right-handed subjects performed planar, centre-out arm reaching movement trials with a robotic manipulandum in three phases: a null force field (baseline), a velocity-dependent force field (adaptation; 25 Nsm-1) and again in a null force field (deadaptation). Reaching error and voluntary EMG were recorded from the biceps and triceps before, during and after motor adaptation. We also explored the effects of motor adaptation on evoked responses to single and paired pulse Transcranial Magnetic Stimulation from the same muscles at different delays after a visual go command, but before the onset of voluntary muscle activity. After the force field was removed, subjects produced reaching overshoot characteristic of adaptive internal model formation. Following motor adaptation, there was a significant increase in corticospinal excitability, reduction in short interval intracortical inhibition and increase in short interval intracortical facilitation that was associated with a sustained increase in voluntary muscle activity in the biceps. The adaptation-driven increase in reaching overshoot coupled with the increase in voluntary activity, corticospinal and intracortical excitability in the biceps suggests that the M1 may specify some of the feed forward components of newly learnt internal models through the control of specific muscles.
\end{abstract}

Keywords: TMS, Internal Model Formation, Corticospinal Excitability

\section{Introduction}

Healthy adults are capable of rapidly adapting to changes in external forces during reaching and grasping. At least two predictive control strategies are thought to contribute to motor adaptation including; increasing the stiffness of the arm through co-contraction of antagonistic muscles in the upper limb $[1,2]$ and by generating a prediction of the expected perturbing forces through internal model formation $[3,4]$.

Whilst the neurological network underlying these control mechanisms has not been fully elucidated, one area that has been implicated in primates is the primary motor cortex (M1).When primates adapt their reaching movements to a force field perturbation, a population of M1 cells alter their firing properties in parallel with the directional changes in muscle activity needed to perform the adapted task $[5,6]$. Invasive neuronal recordings are not practical in healthy adults; nonetheless changes in M1 output to the corticospinal pathways can be measured in the form of motor evoked potentials (MEPs) evoked by Transcranial Magnetic Stimulation (TMS). A number of studies have reported a rapid build up of corticospinal excitability in agonist muscles $20-100 \mathrm{~ms}$ before the onset of voluntary muscle activation during simple and over learnt finger, thumb and upper limb move- 
ments $[7,8]$. Notably, this ramping up of agonist corticospinal excitability before a phasic movement has also been associated with a reduction in the level of short interval intracortical inhibition (SICI) [7,9]. Together these findings suggest that the M1 drives part of initial output of well learnt internal models by specifying patterns of muscle activation, but do not reveal the involvement of the M1 in specifying muscle activation patterns in newly developed internal models under novel dynamic conditions.

The present experiments were designed to extend this previous work by examining the changes in corticomotor excitability associated with newly formed internal models after motor adaptation in healthy adults. One of the most commonly used paradigms to investigate this type of motor learning is force field motor adaptation, which involves subjects making repeated horizontal plane reaching movements with a manipulandum before, during and after the application of an induced force field. The field initially perturbs the movement and increases the curvature of the reaching movement from the ideal straight line trajectory. However, with repeated practice subjects adapt to the forces, and are able to straighten their hand trajectory [10]. The formation of a newly learned internal model has been demonstrated by an initial "overshoot" reaching movement, into the direction of the force field, once the force field has been removed [10]. Thoroughman and Shadmehr (1999) [2], showed that the introduction of a force field during horizontal plane, centre-out, leftward reaching movements $\left(135^{\circ}\right.$ target) can lead to a substantial and sustained increase in voluntary muscle activity in the biceps that was associated with internal model formation. In contrast, there is little modulation of biceps muscle activity during force field adaptation involving reaching towards the body (270 ${ }^{\circ}$ target) [11]. We hypothesized, therefore, that if the M1 was driving adaptation-induced changes of feedforward motor control by directly controlling some of the muscles involved in the activity there would be a significant increase in corticospinal excitability in the biceps, following adaptation to the $135^{\circ}$ target but not during adaptation to the $270^{\circ}$ target.

\section{Methods}

\subsection{Subjects}

Ten healthy right handed adult volunteers (22 - 38 years old; 7 male) [12], participated in the first study after providing informed consent. Eight healthy right handed adults (22 - 38 years old; 5 male) who had previously completed the first study took part in the second study. All experiments were approved by the University of East
London local ethics committee and were conducted in accordance with the Declaration of Helsinki.

\subsection{Motor Adaptation Paradigm}

Motor adaptation took place using a shoulder/arm robotic manipulandum (Interactive Motion Technologies, Cambridge, USA). Subjects sat in a customized chair and grasped the manipulandum with their right hand $\left(70^{\circ}\right.$ shoulder flexion, $90^{\circ}$ elbow flexion, and semi-pronated forearm). The weight of the arm was supported on a plastic forearm prop attached to the manipulandum handle. Centre-out planar reaching movements $(15 \mathrm{~cm}$ path length) were made from a central start position $(1 \mathrm{~cm}$ diameter) to a peripheral target $(1 \mathrm{~cm}$ diameter) in two primary directions 1) towards the body ( $270^{\circ}$ target) and 2) diagonally away from the body $\left(135^{\circ}\right.$ target). Subjects reached to 1 target during one session and the other target during the second session. The sessions were separated by 1 week and the order was counterbalanced. The real time hand position was represented on a vertically oriented computer screen situated at eye level above the manipulandum. Subjects were instructed to move as quickly and accurately as possible from the central start position to the peripheral target when it changed colour from grey to yellow. After reaching the target, subjects were required to maintain the position for a random interval (1.6 - 3.2 secs) before the arm was passively returned to the central start position by the robotic manipulandum. To reduce reaching predictability and main- tain attention, subjects made reaching movements in response to active "Go" targets but not towards "NoGo" targets (Go/NoGo ratio was $70 \%$ ). During each session, subjects performed a total of 240 trials during three phases (10 blocks of 24 trials per block). Initially, 4 blocks were performed in the null force field ( 3 blocks of familiarization with block 4 taken as baseline), followed by 4 blocks in the force field (adaptation phase, blocks 5-8). The forces that acted on the hand during the force field were velocity dependent $\left(25 \mathrm{~N} \cdot \mathrm{ms}^{-1}\right)$ and were applied in a clockwise direction that was orthogonal to the reaching trajectory. Finally, 2 blocks were completed in the null field (deadaptation phase, blocks 9-10).

\subsection{Transcranial Magnetic Stimulation (TMS)}

To investigate changes in corticospinal excitability and levels of excitatory and inhibitory inputs to corticomotor neurons, we used a figure-of-eight coil $(9 \mathrm{~cm}$ outer diameter) connected to two single-pulse, monophasic, Magstim model 200 stimulators through a Bistim module (Magstim Company, Whitland, UK). The Magstim stimulators were triggered using Signal software and CED data 
acquisition interface (Cambridge Electronic Design, Cambridge, UK). The coil was positioned tangentially to the skull with the handle positioned backwards and angled $45^{\circ}$ away from the midline. The optimal coil position ("hot spot") on the scalp for evoking Motor Evoked Potentials (MEPs) in the contralateral biceps brachii muscle was located by applying TMS in $1 \mathrm{~cm}$ steps around the presumed upper limb area. The position of the coil was marked on the subjects' head to ensure consistent placement during each experiment. Resting motor threshold (RMT), was defined as the minimum intensity that induced MEPs $\geq 50 \mu \mathrm{V}$ peak to peak in the biceps in 5 out of 10 trials [13].

\subsection{Single Pulse (SP) TMS}

During the first study TMS measurements were made during 3 testing blocks completed after each paradigm phase (i.e. baseline/adaptation/deadaptation). To characterize the recruitment of the corticospinal pathways before voluntary muscle activation, single pulse TMS was applied at $110 \%$ of RMT pseudo randomly at 5 different time points $(0,130,160,190,220 \mathrm{~ms})$ after a visual go-signal at the active target. All subjects were instructed to 'relax completely' before each trial began and this was confirmed by visual inspection of the EMG signal. In total, 180 trials were completed for the testing blocks (3 blocks of 60 trials). Within each block, there were 10 repeats of each of the 5 TMS time points and 10 control no-TMS trials.

\subsection{Paired Pulsed (PP) TMS}

In the second study, paired-pulse TMS was used to investigate short interval intracortical inhibition (SICI) and intracortical facilitation (ICF) during the 3 testing blocks completed after each paradigm phase. TMS was applied over the biceps representation of the left M1 as in study 1 . For both SICI and ICF, the conditioning stimulus was initially set at $70 \%$ of RMT and the test stimulus was set at $120 \%$ of RMT. SICI was examined using an interstimulus interval (ISI) of $3 \mathrm{~ms}$ (PP3), whilst ICF was examined using an ISI of $13 \mathrm{~ms}$ (PP13) [14]. The intensity of the conditioning stimuli for PP3 and PP13 were adjusted for each subject to ensure that there was significant SICI and ICF respectively at TMS time interval $0 \mathrm{~ms}$. SP MEPs were also evoked by TMS at $120 \%$ RMT. TMS was applied at 3 time points $(0,160,190 \mathrm{~ms})$ after a visual go signal with 3 TMS conditions (SP/PP3/PP13). A total of 360 trials were completed in the three testing blocks (120 trials each block). Within each block, there were 10 trials at each of the 3 time states for each of the 3 TMS conditions (SP/PP13/PP3) and 30 control trials.

\subsection{Data Acquisition and Analysis}

\subsubsection{Movement Kinematics}

Changes in reaching kinematics were recorded by two 16-bit position and velocity encoders in the robot motors. The raw signal was low pass Butterworth-filtered (low pass $30 \mathrm{~Hz}$, high pass $1000 \mathrm{~Hz}$ ), sampled at $200 \mathrm{~Hz}$ and stored on a laptop ready for offline analysis. Motor performance was quantified by two measures of trajectory error in each trial. Summed error ( $\mathrm{SmE})$ is a global movement error calculated as the cumulative perpendicular distance between the hand position and the ideal trajectory (values are positive regardless of path directionality) for the duration of the reaching movement. Signed error $(\mathrm{SgD})$ represents the cumulative perpendicular distance between the hand position and the ideal trajectory over the first $150 \mathrm{~ms}$ of movement, whereby the reaching paths to the right and left of the ideal trajectory were given positive and negative values respectively [15]. It has been suggested that a change in signed error represents formation of an internal model of a new motor task, for example, as the subject predicatively pushes counter-clockwise in order to compensate for the expected clockwise force field resistance [15]. All kinematic measures were referenced to movement onset and movement offset. Movement onset was defined as the time that the hand exceeded a compound $\mathrm{x}-\mathrm{y}$ velocity of $0.03 \mathrm{~m} \cdot \mathrm{s}^{-1}$, whilst movement offset was defined as the time that the hand velocity dropped below $0.03 \mathrm{~m} \cdot \mathrm{s}^{-1}$. Peak velocity was also calculated during each reaching trial to assess global movement performance. All single trial SmE measurements were averaged over the 24 trials of each block. In order to capture the effects of adaptation on internal model formation without including the effects of deadaptation, we compared $\mathrm{SgD}$ on the last trial of baseline with the first trial of deadaptation.

\subsubsection{Muscle Activity}

Surface EMG was recorded from the right biceps and triceps muscles with pre-amplified bipolar surface electrodes with a common reference (Biometrics, Gwent, UK). EMG was band pass filtered at $20 \mathrm{~Hz}$, sampled at 5 $\mathrm{KHz}$, and then stored on a laptop for offline analysis.

\subsubsection{Voluntary EMG}

Prior to analysis, voluntary EMG for each trial was full wave rectified and baseline corrected to resting EMG (average voluntary EMG from $0-100 \mathrm{~ms}$ after the visual go signal). Voluntary muscle activity was quantified as the average EMG, computed from $100 \mathrm{~ms}$ before movement onset to $100 \mathrm{~ms}$ after movement onset. This epoch was chosen because it was considered to include only feed forward commands to muscles [1]. Voluntary EMG 
onset was defined as the time at which voluntary EMG increased by greater than $1 \mathrm{SD}$ of the resting EMG, and this was confirmed by visual inspection. Since previous studies have shown that TMS applied following an external movement cue can alter voluntary response times $[16,17]$, voluntary EMG onsets were measured during the control trials in each test block. All single trial EMG measurements were averaged over the 24 trials of each block. The effects of adaptation and deadaptation on voluntary EMG were measured by comparing changes in EMG in the first and last adaptation blocks (B5 and B8) and final deadaptation block (B10) with EMG in the final baseline block (B4). Previously, it has been reported that velocity dependent force field adaptation occurs rapidly with the largest changes in learning occurring during the first few trials [1]. In order to differentiate initial adaptive versus later adaptive changes in EMG, the reaching blocks were subdivided into bins of 10 individual trials. The last 10 trials of baseline EMG (baseline) were compared with a) the first 10 trials of EMG in the first adaptation block (early adaptation) and b) the last 10 trials in the final adaptation block (late adaptation).

\subsection{TMS Responses}

MEP amplitude was calculated as the peak to peak voltage of the evoked response from TMS. Pre-TMS muscle activity (Pre-EMG) was quantified by measuring the integrated EMG $10 \mathrm{~ms}$ prior to TMS. TMS trials were visually inspected and any which showed evidence of background EMG activity, prior to TMS, were removed from the data set. In order to provide valid comparison for changes in PP responses, these were expressed relative to the appropriate SP values measured at baseline.

Statistical Analyses

Changes in SmE, peak velocity, movement onset and muscle activity were tested for statistical significance with a one way repeated measures ANOVA with reaching block (B4-B10) as the within subject factor. Changes in MEP amplitude were tested for statistical significance with a two way repeated measures ANOVA with testing block (i.e. baseline/adaptation/deadaptation) and TMS time interval $(0,130,160,190,220 \mathrm{~ms})$ as the within subject factors. Post hoc paired t-tests were corrected for multiple comparisons using the Bonferroni correction (2 tailed, $p<0.05)$. All data are shown as mean values \pm standard error (SE).

\section{Results}

Study 1: Effects of adaptation on reaching kinematics.

There were no significant differences in baseline (B4) summed error between the first and second sessions $(p>$ $0.05)$. This indicated that the number of deadaptation blocks used was sufficient to minimize any carryover effects between the two test sessions.

All subjects demonstrated a typical velocity dependent force field adaptation pattern when reaching in both target directions (see Figure 1). There was a significant effect of learning block on SmE (ANOVA: target $135^{\circ}$ :

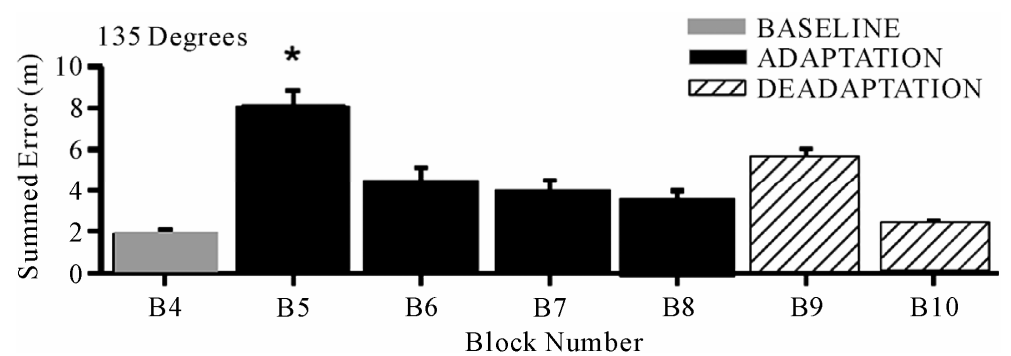

(a)

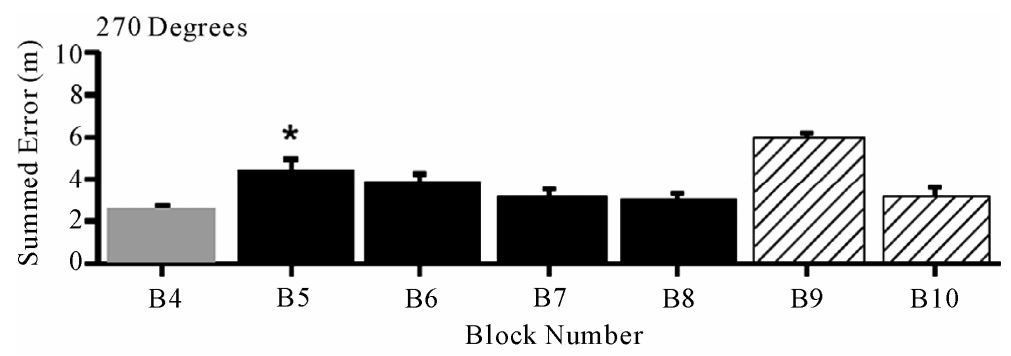

(b)

Figure 1. Summed Error during motor adaptation to the two targets. Mean values $\pm S E(N=10)$ at the $(a) 135^{\circ}$ target and $(b)$ the $270^{\circ}$ target. For both targets there was a significant increase in SmE in the first adaptation block (compared to baseline values) which reduced towards baseline levels over the 4 adaptation blocks $(* p<0.05)$. 
$\mathrm{f}=6.2, p<0.01$; target $\left.270^{\circ}: \mathrm{f}=5.4, p<0.01\right)$. Initially, there was a significant increase in $\mathrm{SmE}$ in the first adaptation block (B5) compared to baseline (B4; target $135^{\circ}$ : $+6.2 \pm 0.6 \mathrm{~m}, p<0.01$; target $270^{\circ}:+1.8 \pm 0.8 \mathrm{~m}, p<$ $0.01)$. As subjects continued reaching in the force field, their accuracy improved as demonstrated by a large reduction in SmE between the first and the last adaptation block (target $135^{\circ}:-4.5 \pm 1.2 \mathrm{~m}, p<0.01$; target $270^{\circ}$ : $-1.3 \pm 0.6 \mathrm{~m}, p<0.01)$. During the first block of deadaptation (B9), there was an initial increase in reaching error into the direction of the force field, which rapidly returned towards baseline values. To analyze whether this "overshoot" represented a modification in the feed forward component of reaching, $\mathrm{SgD}$ during the 1 st movement of deadaptation was compared with that of the last movement at baseline. There was a significant increase in $\mathrm{SgD}$ during the 1 st trial of deadaptation for both targets (target $135^{\circ}$ : $+0.68 \pm 0.2 \mathrm{~m}, p<0.05$; target $270^{\circ}$ : $+1.0 \pm 0.3 \mathrm{~m}, p<0.01)$.

There was no significant effect of reaching block on peak velocity (ANOVA: target $135^{\circ}: f=0.5, p=0.2$; target $270^{\circ}: f=1.4, p=0.11$; or movement onset time (target $135^{\circ}: f=1.2, p=0.1$; target $270^{\circ}: f=1.3, p=$ $0.1)$.

Study 1: Effects of adaptation on voluntary EMG

The average block by block muscle activation patterns during adaptation are shown in Figure 2. Analysis revealed a significant effect of learning block on voluntary EMG in both the biceps (ANOVA: $f=6.6, p<0.01$ ) and triceps (ANOVA $f=5.9, p<0.01$ ) at the $135^{\circ}$ target. Adaptation resulted in two distinctive changes in voluntary EMG at the $135^{\circ}$ target. Firstly, there was a significant increase in EMG amplitude in the biceps $(+0.11 \pm$ $0.03 \mathrm{mV} . \mathrm{ms} ; p<0.01)$ in the first adaptation block. Notably, this increase was sustained at the final adaptation block $(+0.15 \pm 0.01 \mathrm{mV} . \mathrm{ms} ; p<0.05)$ and only returned towards baseline values during deadaptation (Figure 2(a)). Secondly, there was a more gradual reduction in triceps activity during the adaptation blocks that became significant at the final adaptation block $(-0.18 \pm 0.06$ $\mathrm{mV} . \mathrm{ms}, p<0.05)$ and returned to baseline levels during deadaptation (Figure 2(b)).

Within block analysis of early adaptation versus late adaptation in voluntary EMG revealed a third distinctive pattern of EMG modulation at the $270^{\circ}$ target (Figures 2(c) and (d)). There was a significant increase in muscle activity in the biceps and triceps during early adaptation (biceps: $+0.27 \pm 0.16 \mathrm{mV} . \mathrm{ms}, p<0.01$; triceps: $+0.3 \pm$ $0.11 \mathrm{mV} . \mathrm{ms}, p<0.01$ ), that returned towards baseline during late adaptation (biceps: $+0.13 \pm 0.06 \mathrm{mV} . \mathrm{ms}, p>$ 0.05 , triceps: $0.01 \pm 0.04 \mathrm{mV} . \mathrm{ms}, p>0.05$ ).

There were no significant differences in the initial EMG onset for the biceps and triceps at either target
(Table 1). Moreover, there were no significant changes in EMG onset in the any of the muscles during the three experimental phases at the two targets.

Study 1: Effects of adaptation on SP TMS responses.

During movements to the $135^{\circ}$ target, there was a significant time interval $x$ testing block interaction on MEP amplitude measured at the biceps (ANOVA: $f=4.1, \mathrm{p}<$ $0.05)$. Following adaptation in this direction there was a clear trend for biceps MEPs to increase closer to movement onset time, (Figure 3(a)), and these proved significantly different from baseline at $190 \mathrm{~ms}(+0.33 \pm$ $0.04 \mathrm{mV}, p<0.01)$ and $220 \mathrm{~ms}(+0.49 \pm 0.13 \mathrm{mV}, p<$ 0.01 ) after the go signal. However, the MEP amplitude/ time response curve returned towards baseline levels following deadaptation. In contrast, there were no significant changes in triceps MEP responses during learning and deadaptation in this direction (Figure 3(b)).

For the other learning direction $\left(270^{\circ}\right.$ target $)$, there was a tendency for biceps MEPs to increase in amplitude closer to movement onset (Figure 3(c)). This trend showed no change in relation to the adaptation or deadaptation phases. The triceps MEP responses showed no variation in response to learning and deadaptation to the $270^{\circ}$ target (Figure $3(\mathbf{d})$ ).

There was also no effect of the testing block on integrated pre-EMG to either the $135^{\circ}$ (ANOVA biceps: $f=$ $0.8, p=0.4$; triceps: $f=1.3, p=0.2$ ) or $270^{\circ}$ target (ANOVA biceps: $f=2.1, \mathrm{p}=0.1$; triceps: $f=0.9, p=$ 0.3 ). In total, $8 \%$ of trials at the $135^{\circ}$ target and $9 \%$ of trials at the $270^{\circ}$ target were discarded because of pre -EMG. The percentage of disregarded trials was not affected by testing block to either the $135^{\circ}$ (ANOVA: $f=$ $0.9, p=0.32$ ) or the $270^{\circ}$ target (ANOVA: $f=0.7, p=$ $0.4)$.

Study 2: Effects of adaptation on PP TMS responses.

Figures 4 and 5 show the changes in paired pulse responses of the biceps and triceps in response to adaptation and deadaptation at the $135^{\circ}$ target. In the case of PP3 measured at the biceps, there was little change in the MEP amplitude relative to movement onset. However, there was a significant time interval $\mathrm{x}$ testing block interaction (ANOVA: $f=4.1, p<0.05$ ). After adaptation, there was a striking augmentation of PP3 MEPs with a tendency to be more pronounced nearer to movement onset time. This proved to be significant $190 \mathrm{~ms}$ after the visual go signal relative to corresponding baseline values with a gain of $2.46 \pm 0.7(p<0.05)$. Following deadaptation, PP3 MEP amplitude returned towards baseline leading to a gain of $0.85 \pm 0.28(p>0.05)$. PP13 MEPs measured at the biceps showed a similar pattern of facilitation. Following adaptation, there was a large increase in MEP amplitude $160 \mathrm{~ms}$ post go signal relative to baseline with a gain of $3.2 \pm 0.6(p<0.05)$. These val- 


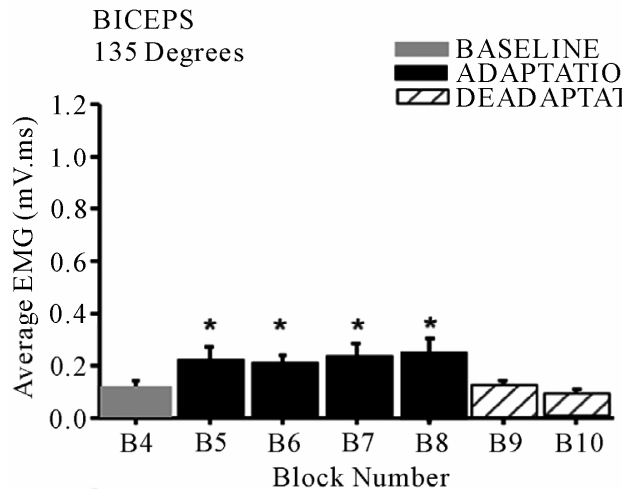

(a)

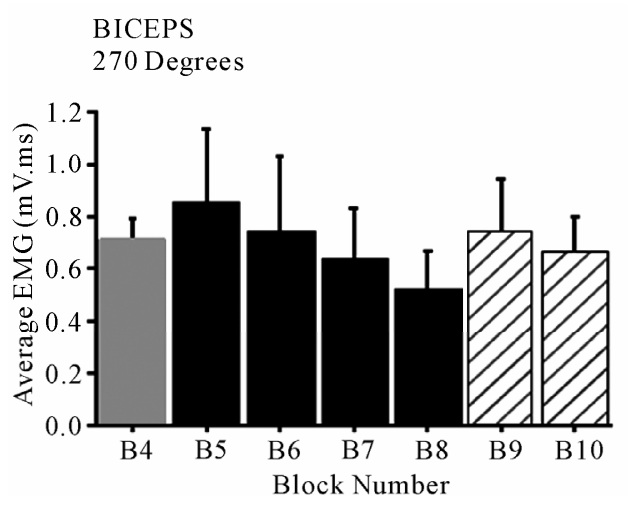

(c)
TRICEPS

135 Degrees

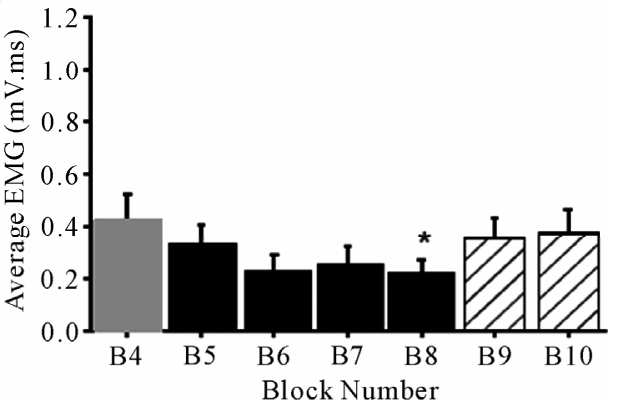

(b)

TRICEPS

270 Degrees

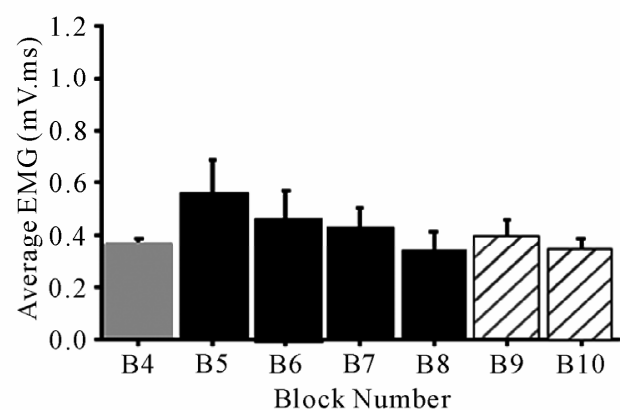

(d)

Figure 2. Muscle activation patterns for the biceps and triceps during motor adaptation at the two targets. Average EMG values $\pm S E(N=10)$. (a) biceps activation to the $135^{\circ}$ target (b) triceps activation to the $270^{\circ}$ target (c) biceps activation to the $270^{\circ}$ target (d) triceps activation to $270^{\circ}$ target. For the $135^{\circ}$ target there was significant and sustained increase in biceps activation during adaptation blocks (B5-B8) and a significant reduction in triceps activity during the last 3 adaptation blocks (B6-B8) compared to baseline (B4).

Table 1. Mean EMG onsets for the biceps and the triceps during the 3 experimental phases of the experiment. The table shows mean EMG onsets \pm SE $(\mathrm{N}=10)$. There was no effect of testing block on EMG onset in either muscle at either target.

\begin{tabular}{ccccccc}
\hline \multirow{2}{*}{ Muscle } & \multicolumn{2}{c}{ EMG onset $\mathbf{1 3 5}^{\circ}$ Target $(\mathbf{m s} \pm$ SE) } & \multicolumn{2}{c}{ EMG onset $270^{\circ}$ Target $(\mathbf{m s} \pm$ SE) } \\
\cline { 2 - 7 } & Baseline & Adaptation & Deadaptation & Baseline & Adaptation & Deadaptation \\
\hline Biceps & $261 \pm 10$ & $263 \pm 10$ & $267 \pm 12$ & $252 \pm 14$ & $255 \pm 16$ & $246 \pm 16$ \\
Triceps & $248 \pm 12$ & $250 \pm 14$ & $252 \pm 12$ & $251 \pm 12$ & $259 \pm 15$ & $249 \pm 22$ \\
\hline
\end{tabular}

ues reverted towards baseline following deadaptation with a gain of $2.3 \pm 0.6(p>0.05)$.

There was no significant effect of the testing block on integrated pre-EMG to the $135^{\circ}$ target (ANOVA biceps: $f$ $=0.6, p=0.5$; triceps: $f=0.8, p=0.4)$.

\section{Discussion}

\subsection{Effects of Adaptation on Reaching Kinematics}

The significant reduction in performance error demon- strated during adaptation to both targets suggests that subjects had learnt to adapt to the force fields. This was expected, since healthy adults have been shown to rapidly adapt to repeated exposure of a velocity dependent force field $[1,10]$. One way in which subjects are thought to adapt is by predicatively compensating for perturbation forces using an internal representation of the expected dynamics (internal model) [1,2]. All subjects in this study demonstrated an increase in $\mathrm{SgD}$ during the first trial of deadaptation, suggesting that subjects had developed a prediction of the expected dynamics after adaptation at both targets. 


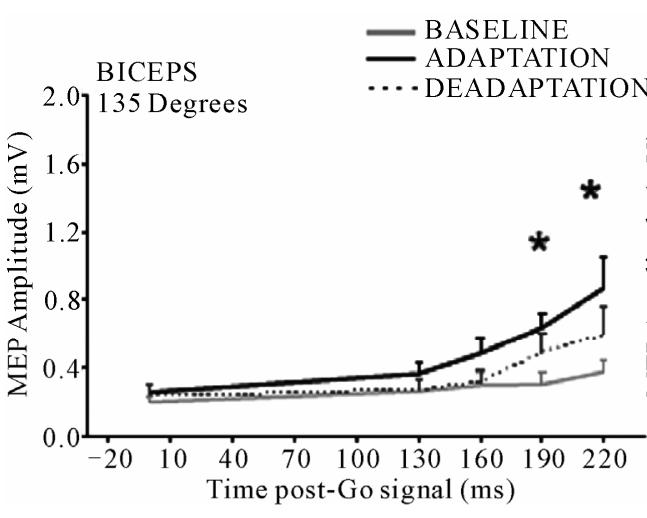

(a)

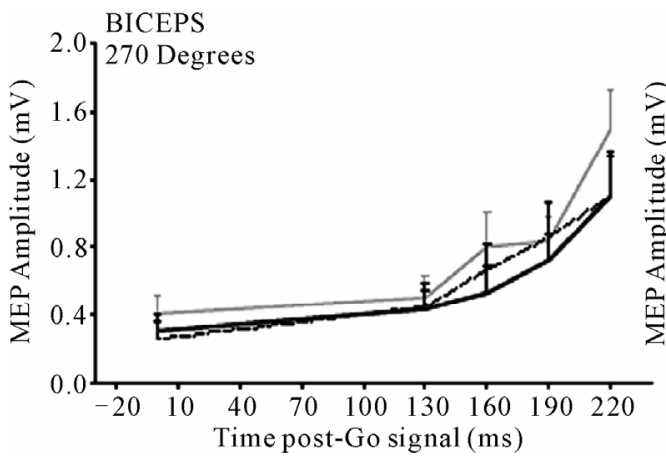

(c)

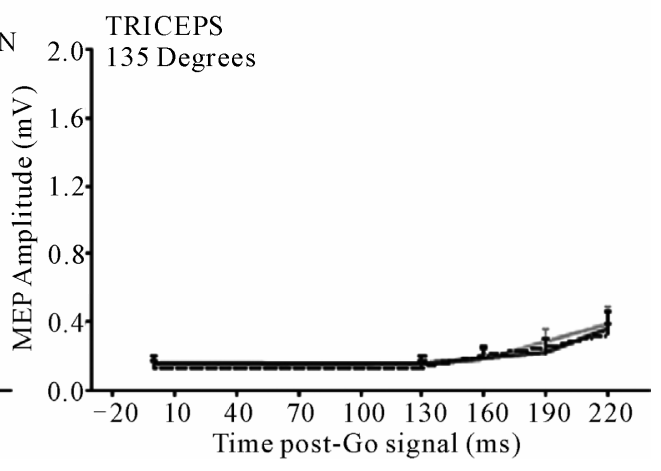

(b)

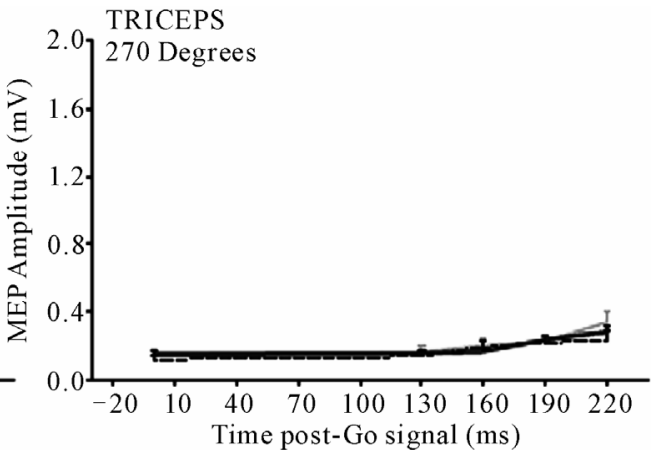

(d)

Figure 3. MEP amplitude patterns for the biceps and triceps during motor adaptation at the two targets. Average MEP amplitude $\pm \mathrm{SE}(\mathrm{N}=10)$ for (a) Biceps at the $135^{\circ}$ target (b) triceps at the $270^{\circ}$ target (c) biceps at the $270^{\circ}$ target (d) triceps at the $270^{\circ}$ target. During adaptation at the $135^{\circ}$ target there was a significant increase in MEP amplitude in the biceps 190 and $220 \mathrm{~ms}$ after the visual go signal compared to corresponding baseline values $(* p<0.05)$.

\subsection{Effects of Adaptation on Voluntary EMG}

In agreement with previous findings, there was an early and sustained increase in voluntary activity in biceps during adaptation at the $135^{\circ}$ target [2]. This was not simply a load dependent modulation, because it did not occur when subjects adapted at the $270^{\circ}$ target. Previous studies have suggested that increased activity in antagonistic upper limb muscles may be used to increase arm stability during motor adaptation $[1,18]$. Commonly, however, muscle activity initially increases and subsequently shows a gradual decline during repeated exposure to a velocity dependent force field $[18,19]$. This pattern of activity does not resemble the sustained elevation of voluntary activity in the biceps throughout the adaptation blocks. Whilst we cannot rule out some element of cocontraction, the continued increase in biceps activity is more characteristic of a muscle being recruited to predicatively counteract a force field as part of an internal model strategy $[1,2]$. We did not measure torque levels at the elbow and shoulder joint during this study. However, as the primary muscles of elbow flexion and extension, the increase in biceps activity and reduction in triceps activity observed are likely to have resulted in an increased flexor torque at the elbow, which would account for the reaching overshoot observed after force field removal.

In contrast to the findings at the $135^{\circ}$ target, there was an early increase in biceps and triceps activity during adaptation to the $270^{\circ}$ target that gradually reduced over the adaptation blocks. In this case, increased agonist antagonist co-contraction may have been used during the early stages of adaptation to improve stability [18].

\subsection{Effects of Adaptation on Corticospinal Excitability}

After adaptation at the $135^{\circ}$ target, corticospinal excitability measured from the biceps significantly increased at 190 and $220 \mathrm{~ms}$ after the visual go signal. Although there were insufficient time points to accurately identify the onset of these changes in corticospinal excitability in relation to voluntary EMG onset, it was clear that the post-adaptation changes occurred pre-voluntary activation, and thus were feed forward in nature. An increase in corticospinal excitability just before voluntary EMG is 
thought to represent a mechanism by which the M1 drives the initial burst of muscle activity in the agonist $[20,21]$. It is notable that the excitability changes following adaptation reversed after removal of the forcefield (deadaptation). We suggest that this may reflect an adaptation driven change in the feed forward control of the biceps. This is supported by the fact that the raised excitability was associated with a significant increase in voluntary activation in the biceps. Secondly, no changes in MEP amplitude were evident following adaptation to the $270^{\circ}$ target, when EMG was not significantly altered after learning.

When interpreting these results, it is important to rule out other factors that could modulate corticospinal excitability, such as an increased level of pre-movement muscle activation during the adaptation phase [22]. This is unlikely to account for the results in the current study since we carefully monitored EMG activity, and all trials with pre-TMS EMG activity were removed from analysis. Furthermore, there was no change in pre-TMS EMG activity over the 3 testing blocks. Corticospinal excitability increases sharply prior to voluntary muscle activation [7] and a learning induced shortening of the biceps EMG onset could have also increased MEP amplitude in the later TMS states. However, there was no trend for reduced reaction time in the biceps between the 3 testing phases making this possibility unlikely.

Despite the reduction in voluntary EMG in the triceps during adaptation to the $135^{\circ}$ target, there was no significant reduction in corticospinal excitability in the triceps. The lack of change in corticospinal excitability raises the question as to whether adaptive changes in muscle activity may be driven through different neural pathways for upper limb flexors and extensors. This idea is supported indirectly by studies which highlight differences between the corticospinal projections to upper limb flexors and the upper limb extensors [23,24]. Palmer and Ashby (1992) [24] found that TMS over the M1 induced greater excitatory post synaptic potentials in the biceps compared to the triceps. TMS is thought to predominantly activate monosynaptic corticospinal pathways [25] suggesting stronger and more numerous monosynaptic excitatory connections to the biceps compared to the triceps. These findings are mirrored in results of Phillips and Porter (1964) [23], who demonstrated greater monosynaptic facilitation in motoneurons of elbow flexors compared to elbow extensors in baboons.

Corticospinal excitability reflects the net effect of intracortical and spinal facilitatory/inhibitory circuits on the corticospinal pathways. The results from single pulse MEPs, therefore, cannot determine if the adaptation driven changes in excitability occurred at the level of the M1. In the second study, a paired pulse TMS paradigm was used to address this issue.

\subsection{Effects of Adaptation on Intracortical Excitability}

The present findings demonstrate a time dependent modification in SICI and ICF in the biceps after adaptation of reaching to the $135^{\circ}$ target. These changes were not due to an increase in pre-movement muscle activation because trials with pre-TMS EMG activity were excluded. In addition, there were no differences in EMG onset times of the biceps following adaptation.

It has been suggested that SICI originates within the primary motor cortex [26] and is mediated by changes in $\gamma$-aminobutyric acid (GABA) receptor A activity [27,28]. Several studies on human subjects have also shown reduction in SICI in agonist muscles before voluntary EMG onset [7,9], and we observed a trend for reduced SICI in both the biceps and triceps during baseline the nearer to movement onset that TMS was delivered (Figures 4(a) and 5(a)). This may serve to focus excitatory drive to key muscles involved in a specific movement [29]. Notably, following adaptation, there was a dramatic reduction in the level of SICI for the later TMS time point pre-movement in the biceps, but not the triceps. A decrease in the level of intracortical inhibition following motor training of the ankle dorsiflexors has been reported previously [30], and our findings are consistent, despite the fact that we used a proximal upper limb muscle and a more complex dynamic sensorimotor learning task. Moreover, our results support the concept that modulation of SICI plays a role in the process of cortical plasticity, associated with internal model formation during motor skill learning. Further evidence for the role of GABA receptor activity in motor learning in humans has been provided by in vivo magnetic spectroscopy studies. Floyer-Lea et al. (2006) [31] found a 20\% reduction in GABA concentration (consistent with reduced GABAinhibition) following finger sequence learning which was specific to the learning task and motor cortical region of the active hand.

The facilitation produced by a paired pulse paradigm with a sub-threshold S1 and interstimulus interval 10 - 15 $\mathrm{ms}$ (ICF) is less well characterised than SICI, but is also believed to be intracortical in nature. Based on pharmacological studies, it is thought that ICF is primarily related to glutamatergic receptor activity, but may also be influenced by the level of GABAergic inhibition [32]. Thus, although ICF may have a different mechanism from SICI, they may show some interactions. We found that ICF of the biceps showed a similar pattern of increase in association with motor adaptation to the decreases observed in SICI over the same time periods 


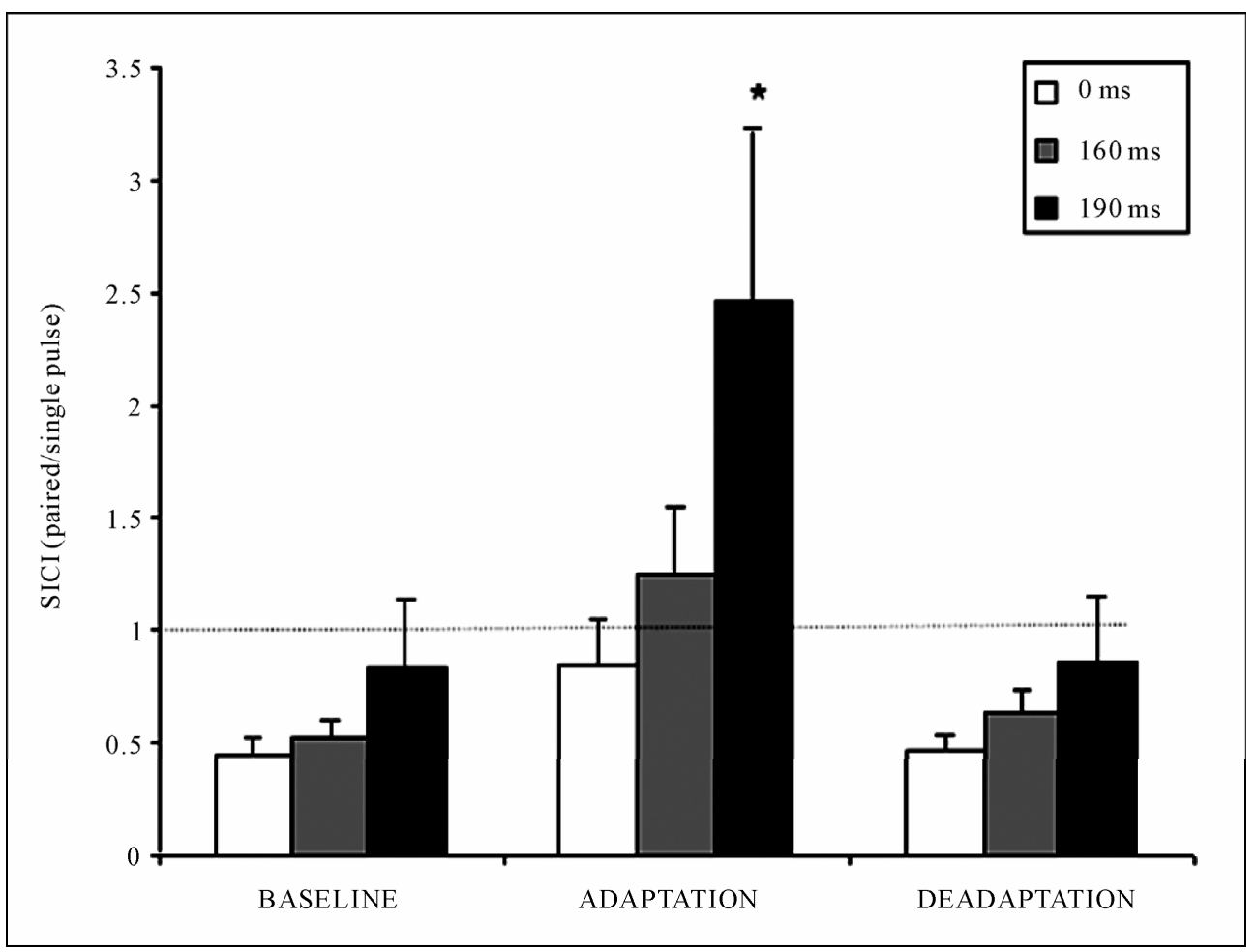

(a)

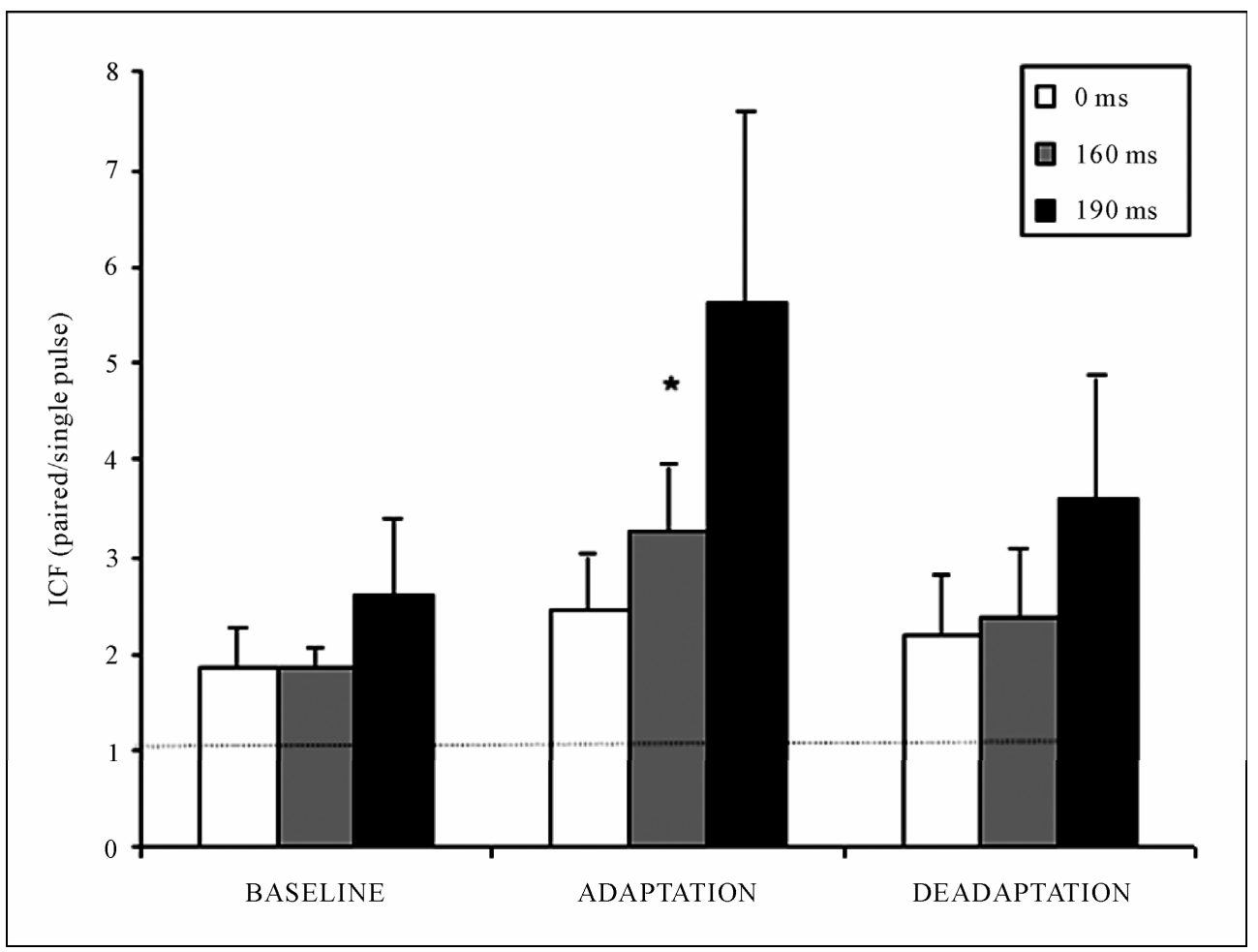

(b)

Figure 4. Mean paired pulse to single pulse MEP ratios for the biceps brachii during learning movements in the $135^{\circ}$ direction (+ SEM). (a) = short-interval intracortical inhibition at ISI $3 \mathrm{~ms}$ (SICI); (b) = intracortical facilitation at ISI $13 \mathrm{~ms}$ (ICF). A significant decrease in SICI occurred following adaptation at $190 \mathbf{~ m s}$ after the visual go signal, and a significant increase in ICF took place at the $160 \mathrm{~ms}$ post go time point $\left({ }^{*} p<0.05\right.$ compared to baseline). 


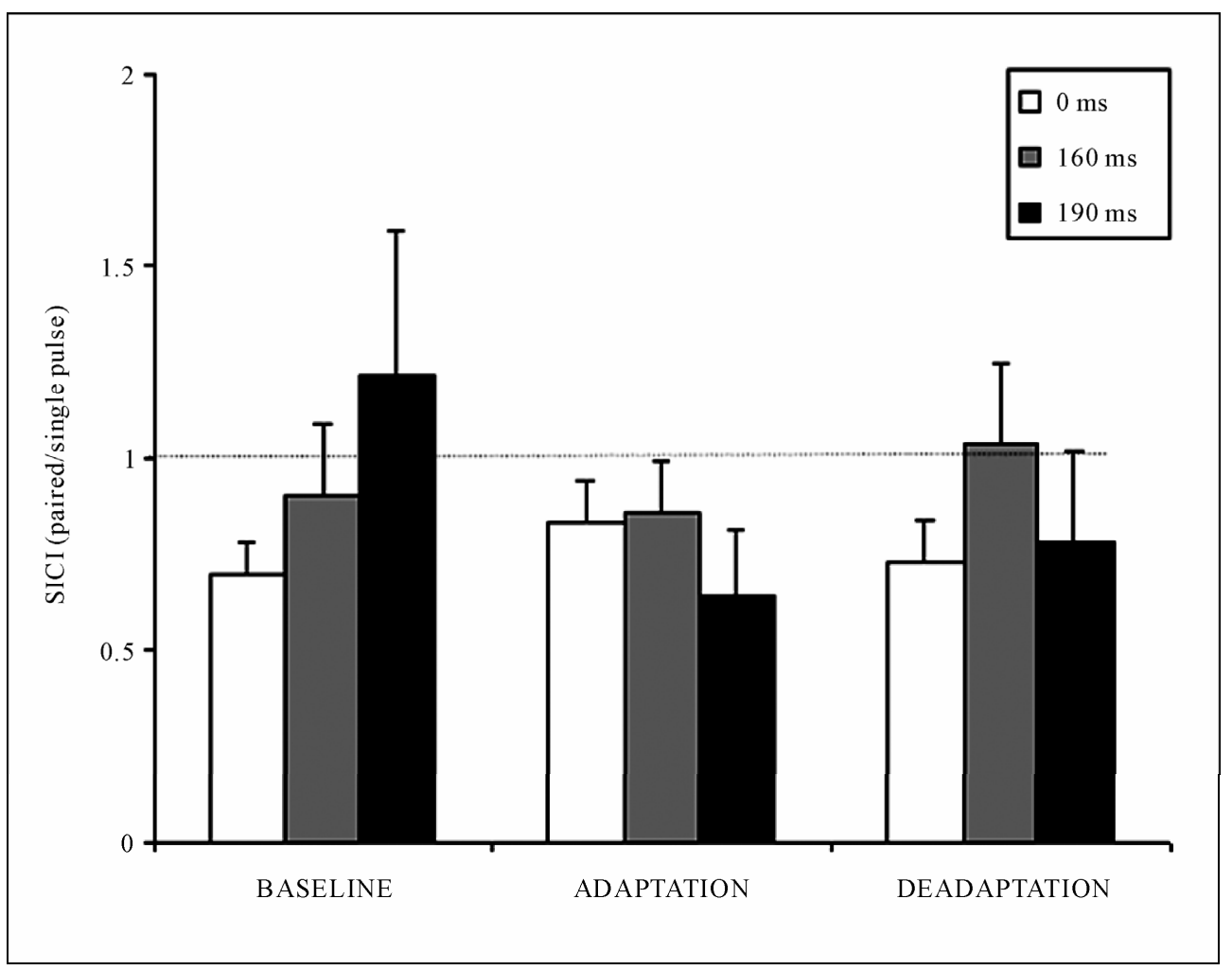

(a)

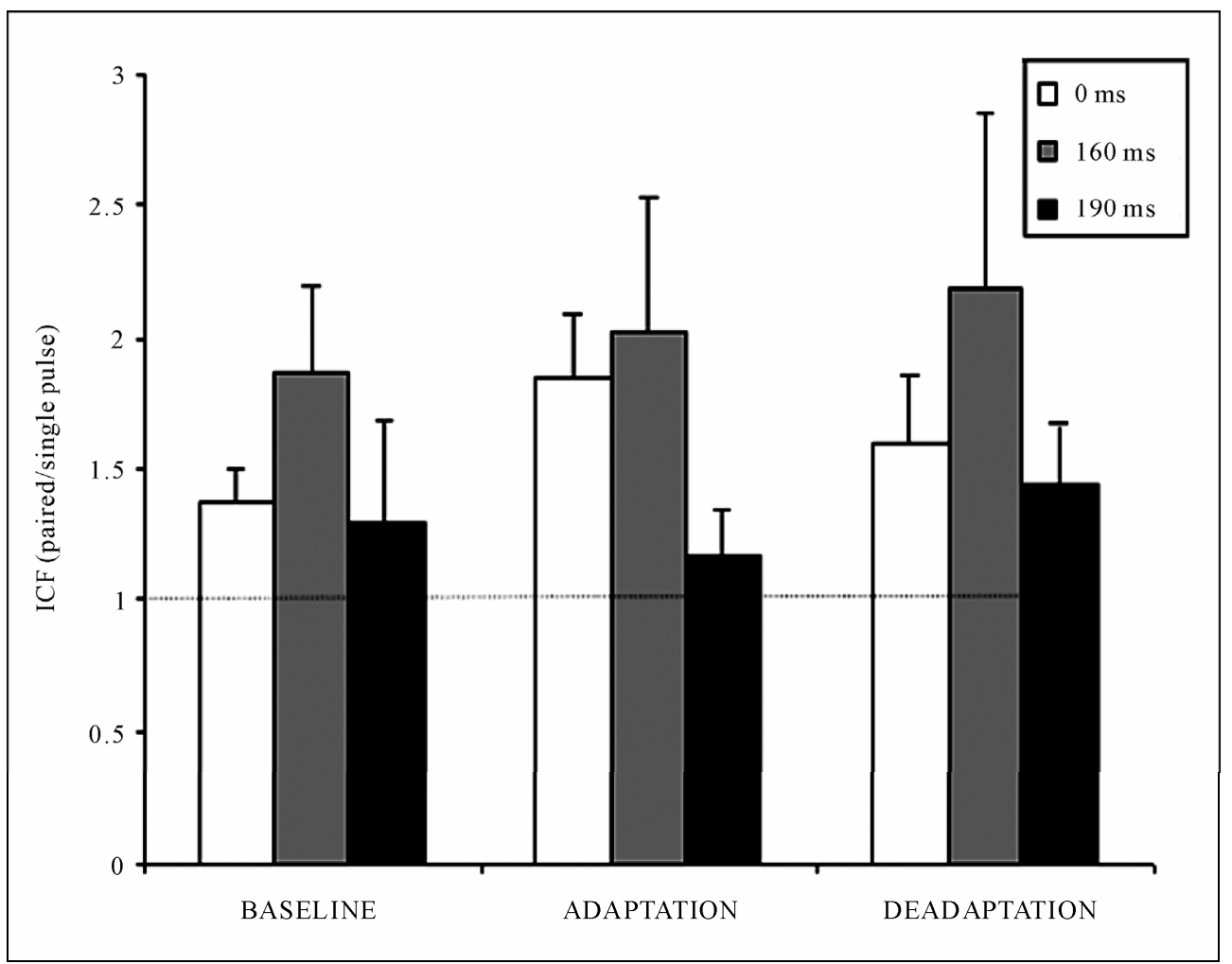

(b)

Figure 5. Mean paired-pulse to single pulse MEP ratios for the triceps brachii during learning movements in the $135^{\circ}$ direction (+ SEM). (a) = short-interval intracortical inhibition at ISI $3 \mathrm{~ms}$ (SICI); (b) = intracortical facilitation at ISI $13 \mathrm{~ms}$ (ICF). 
(Figure 4). This is in contrast to results reported by Liepert et al. (1998) [33] who showed smaller changes in ICF relative to SICI associated with a timed thumb abduction task. The difference may well reflect the differences in the number of muscle groups used and complexity of the learning task between studies.

One of the limitations of using paired-pulse TMS in this type of study is that the non conditioned MEPs can change just before voluntary EMG onset, which hampers the comparison of paired pulse MEP responses at different time states. Considering the dynamic nature of the task, it would have been difficult to have matched MEPs across strict time intervals. Despite this, several studies have shown that adjusting TMS intensity to account for changes, test MEP size has no effect on the level of inhibition when the test MEPs ranged between 1 and $4 \mathrm{mV}$ $[34,35]$.

The majority of reported studies of motor learning have involved simple over learnt hand and wrist tasks and less is known about the role of SICI and ICF in controlling more complex ballistic upper limb movements in humans. Nonetheless, our findings are indirectly supported by adaptation studies on primates, which have reported a force-field induced shift in the directional tuning of small populations of M1 cells that correlated with changes in the directional tuning of upper limb muscles $[6,36]$.

Whilst few studies have looked at the effects of force field adaptation on corticospinal excitability, an alternative approach to investigate the role of the M1 in this form of learning has been to alter M1 excitability using neuromodulation prior to performance of the learning task. Hunter et al. (2009) [37] applied Transcranial Direct Current Stimulation over the M1 during motor adaptation with parameters designed to increase corticospinal excitability. In contrast Hadipour-Niktarash et al. (2007) [38] applied rtms before motor adaptation, using parameters known to decrease corticospinal excitability. Most notably, neither paradigm had a noticeable effect on the control of movement during adaptation. One interpretation is that the M1 is not important in the execution of movement. This seems unlikely given that previous primate studies [6,36] and the current study have all demonstrated changes in both motor performance and activity in the M1 during motor adaptation. A more plausible alternative is that the changes in excitability were not large enough to interfere with motor function or that other brain areas such as the SMA and PMA area could compensate for changes in M1 excitability [5,39].

It is noteworthy that whilst these findings support the idea that the M1drives part of initial output of newly formed internal models by specifying patterns of muscle activation, it does not rule out other coding parameters at the M1.

Previous research on hierarchical cortical processing has identified that neurons in the M1 relate to both abstract movement parameters, such as movement direction and movement speed, as well as muscle activity [40]. Furthermore, there is growing evidence that different movement parameters may be mediated by different descending pathways [41]. There is a general assumption that short-latency MEP responses evoked by TMS are mediated by fast corticospinal neurons with monosynaptic connections [25]. Other movement parameters may be mediated by indirect corticospinal pathways, which would enable more abstract motor commands to undergo additional processing at the spinal level. Yanai et al. (2008) [42] compared firing patterns of a subset of M1 neurons, spinal interneurones and muscles. Notably, they found that the spinal interneurones followed more of a muscle-based pattern of firing compared to the neurons in the M1, which suggests that for some control signals the final stage of muscle based coding may take place at a spinal level.

\section{Conclusions}

The aim of the current study was to investigate the role of the M1 in controlling the output of newly formed internal models in healthy adults. The present study yielded three main results. Firstly, subjects demonstrated reaching overshoot characteristic of internal model formation after adaptation to both targets. Secondly, there was an adaptation dependent sustained increase in feed forward voluntary muscle activity in the biceps after adaptation to the $135^{\circ}$ target. Thirdly, these changes were associated with a temporally dependent increase in single/paired pulse MEP amplitude in the biceps. The initial command to generate well learnt upper limb movements is thought to be mediated by the M1. The present results are consistent with the idea that M1 also drives initial output of newly developed internal models. Moreover, part of this feed forward signal is likely to be directly controlling some of the muscles involved in the activity. Finding an association between intracortical excitability and voluntary muscle activity does not exclude the possibility that the M1 codes other movement parameters. Indeed, there is growing evidence that the M1 can code multiple movement control parameters through different descending pathways.

\section{References}

[1] T. E. Milner and D. W. Franklin, "Impedance Control and Internal Model Use during the Initial Stage of Adaptation to Novel Dynamics in Humans," The Journal of 
Physiology, Vol. 567, 2005, pp. 651-664. doi:10.1113/jphysiol.2005.090449

[2] K. A. Thoroughman and R. Shadmehr, "Electromyographic Correlates of Learning an Internal Model of Reaching Movements," The Journal of Neuroscience, Vol. 19, No. 19, 1999, pp. 8573-8588.

[3] D. M. Wolpert, Z. Ghahramani and M. I. Jordan, “An Internal Model for Sensorimotor Integration," Science, Vol. 269, No. 5232, 1995, pp. 1880-1882. http://dx.doi.org/10.1126/science.7569931

[4] D. M. Wolpert and Z. Ghahramani, "Computational Principles of Movement Neuroscience," Nature Neuroscience, Vol. 3, 2000, pp. 1212-1217. doi:10.1038/81497

[5] C. Padoa-Schioppa, C. S. Li and E. Bizzi, "Neuronal Correlates of Kinematics-to-Dynamics Transformation in the Supplementary Motor Area," Neuron, Vol. 36, No. 4, 2002, pp. 751-765. doi:10.1016/S0896-6273(02)01028-0

[6] C. S. Li, C. Padoa-Schioppa and E. Bizzi, "Neuronal Correlates of Motor Performance and Motor Learning in the Primary Motor Cortex of Monkeys Adapting to an External Force Field," Neuron, Vol. 30, No. 2, 2001, pp. 593607. doi:10.1016/S0896-6273(01)00301-4

[7] M. Nikolova, N. Pondev, L. Christova, W. Wolf and A. R. Kossev, "Motor Cortex Excitability Changes Preceding Voluntary Muscle Activity in Simple Reaction Time Task," European Journal of Applied Physiology, Vol. 98, No. 2, 2006, pp. 212-219. doi:10.1007/s00421-006-0265-y

[8] C. D. MacKinnon and J. C. Rothwell, "Time-Varying Changes in Corticospinal Excitability Accompanying the Triphasic EMG Pattern in Humans," The Journal of Physiology, Vol. 528, No. 3, 2000, pp. 633-645. doi:10.1111/j.1469-7793.2000.00633.x

[9] E. Palmer, E. Cafarelli and P. Ashby, "The Processing of Human Ballistic Movements Explored by Stimulation over the Cortex," The Journal of Physiology, Vol. 481, No. 2, 1994, pp. 509-520.

[10] R. Shadmehr and F. A. Mussa-Ivaldi, "Adaptive Representation of Dynamics during Learning of a Motor Task," The Journal of Neuroscience, Vol. 14, No. 5, 1994, pp. 3208-3224.

[11] T. Hunter, P. Sacco and D. L. Turner, "Corticospinal and EMG Correlates of Internal Model Formation during Force Field Motor Adaptation," Society for Neuroscience Annual Meeting, 2008.

[12] R. C. Oldfield, "The Assessment and Analysis of Handedness: The Edinburgh Inventory," Neuropsychologia, Vol. 9, No. 1, 1971, pp. 97-113. doi:10.1016/0028-3932(71)90067-4

[13] P. M. Rossini, A. T. Barker, A. Berardelli, M. D. Caramia, G. Caruso, R. R. Cracco and M. R. Dimitrijevic, "NonInvasive Electrical and Magnetic Stimulation of the Brain, Spinal Cord and Roots: Basic Principles and Procedures for Routine Clinical Application. Report of an Ifcn Committee," Electroencephalography and Clinical Neurophysiology, Vol. 91, No. 2, 1994, pp. 79-92. doi:10.1016/0013-4694(94)90029-9
[14] A.R. Kossev, S. Siggelkow, R. Dengler and J. D. Rollnik, "Intracortical Inhibition and Facilitation in Paired-Pulse Transcranial Magnetic Stimulation: Effect of Conditioning Stimulus Intensity on Sizes and Latencies of Motor Evoked Potentials," Journal of Clinical Neurophysiology, Vol. 20, No. 1, 2003, pp. 54-58. doi:10.1097/00004691-200302000-00007

[15] R. Osu, E. Burdet, D. W. Franklin, T. E. Milner and M. Kawato, "Different Mechanisms Involved in Adaptation to Stable and Unstable Dynamics," Journal of Neurophysiology, Vol. 90, No. 5, 2003, pp. 3255-3269. doi:10.1152/jn.00073.2003

[16] S. McMillan, V. Nougier and W. D. Byblow, "Human Corticospinal Excitability during a Precued Reaction Time Paradigm," Experimental Brain Research, Vol. 156, No. 1, 2004, pp. 80-87. doi:10.1007/s00221-003-1772-2

[17] B. L. Day, J. C. Rothwell, P. D. Thompson, N. A. Maertens, K. Nakashima, K. Shannon and C. D. Marsden, "Delay in the Execution of Voluntary Movement by Electrical or Magnetic Brain Stimulation in Intact Man. Evidence for the Storage of Motor Programs in the Brain," Brain, Vol. 112, No. 3, 1989, pp. 649-663. doi:10.1093/brain/112.3.649

[18] R. Osu, D. W. Franklin, H. Kato, H. Gomi, K. Domen, T. Yoshioka and M. Kawato, "Short- and Long-Term Changes in Joint Co-Contraction Associated with Motor Learning as Revealed from Surface EMG," Journal of Neurophysiology, Vol. 88, No. 2, 2002, pp. 991-1004.

[19] D. W. Franklin, R. Osu, E. Burdet, M. Kawato and T. E. Milner, "Adaptation to Stable and Unstable Dynamics Achieved by Combined Impedance Control and Inverse Dynamics Model," Journal of Neurophysiology, Vol. 90, No. 5, 2003, pp. 3270-3282. doi:10.1152/jn.01112.2002

[20] C. Reynolds and P. Ashby, "Inhibition in the Human Motor Cortex Is Reduced Just before a Voluntary Contraction," Neurology, Vol. 53, No. , 1999, pp. 730-735.

[21] W. Muellbacher, U. Ziemann, B. Boroojerdi, L. Cohen and M. Hallett, "Role of the Human Motor Cortex in Rapid Motor Learning," Experimental Brain Research, Vol. 136, No. 4, 2001, pp. 431-438. doi: $10.1007 / \mathrm{s} 002210000614$

[22] C. L. Lim and C. Yiannikas, "Motor Evoked Potentials: A New Method of Controlled Facilitation Using Quantitative Surface EMG," Electroencephalography and Clinical Neurophysiology, Vol. 85, No. 1, 1992, pp. 38-41. doi:10.1016/0168-5597(92)90099-W

[23] C. G. Phillips and R. Porter, "The Pyramidal Projection to Motoneurones of Some Muscle Groups of the Baboon's Forelimb," Progress in Brain Research, Vol. 12, 1964, pp. 222-245. doi:10.1016/S0079-6123(08)60625-1

[24] E. Palmer and P. Ashby, "Corticospinal Projections to Upper Limb Motoneurones in Humans," The Journal of Physiology, Vol. 448, 1992, pp. 397-412.

[25] J. C. Rothwell, P. D. Thompson, B. L. Day, S. Boyd and, C. D. Marsden, "Stimulation of the Human Motor Cortex through the Scalp," Experimental Physiology, Vol. 76, No. 2, 1991, pp. 159- 200.

[26] J. Valls-Sole, A. Pascual-Leone, E. M. Wassermann and 
M. Hallett, "Human Motor Evoked Responses to Paired Transcranial Magnetic Stimuli," Electroencephalography and Clinical Neurophysiology, Vol. 85, No. 6, 1992, pp. 355-364. doi:10.1016/0168-5597(92)90048-G

[27] T. Kujirai, M. D. Caramia, J. C. Rothwell, B. L. Day, P. D. Thompson, A. Ferbert, S. Wroe, P. Asselman and C. D. Marsden, "Corticocortical Inhibition in Human Motor Cortex," The Journal of Physiology, Vol. 471, No. 1, 1993, pp. 501-519.

[28] V. Di Lazzaro, A. Oliviero, P. Profice, A. Insola, P. Mazzone, P. Tonali and J. C. Rothwell, "Direct Recordings of Descending Volleys after Transcranial Magnetic and Electric Motor Cortex Stimulation in Conscious Humans," Electroencephalography and Clinical Neurophysiology, Supplement, Vol. 51, 1999, pp. 120-126.

[29] M. C. Ridding, J. L. Taylor and J. C. Rothwell, "The Effect of Voluntary Contraction on Cortico-Cortical Inhibition In Human Motor Cortex," The Journal of Physiology, Vol. 487, No. 2, 1995, pp. 541-548.

[30] M. A. Perez, B. K. Lungholt, K. Nyborg and J. B. Nielsen, "Motor Skill Training Induces Changes in the Excitability of the Leg Cortical Area in Healthy Humans," Experimental Brain Research, Vol. 159, No. 2, 2004, pp. 197205. doi:10.1007/s00221-004-1947-5

[31] A. Floyer-Lea, M. Wylezinska, T. Kincses and P. M. Matthews, "Rapid Modulation of GABA Concentration in Human Sensorimotor Cortex during Motor Learning," Journal of Neurophysiology, Vol. 95, No. 3, 2006, pp. 1639-1644. doi:10.1152/jn.00346.2005

[32] U. Ziemann, "Pharmacology of TMS," Supplements to Clinical Neurophysiology, Vol. 56, 2003, pp. 226-231. doi:10.1016/S1567-424X(09)70226-0

[33] J. Liepert, J. Classen, L. G. Cohen and M. Hallett, "TaskDependent Changes of Intracortical Inhibition," Experimental Brain Research, Vol. 118, No. 3, 1998, pp. 421426. doi:10.1007/s002210050296

[34] Z. J. Daskalakis, B. K. Christensen, R. Chen, P. B. Fitzgerald, R. B. Zipursky and S. Kapur, "Evidence for Impaired Cortical Inhibition in Schizophrenia Using Transcranial Magnetic Stimulation," Archives of General Psychiatry, Vol. 59, 2002, pp. 347-354. doi:10.1001/archpsyc.59.4.347
[35] L. Roshan, G. O. Paradiso and R. Chen, "Two Phases of Short-Interval Intracortical Inhibition," Experimental Brain Research, Vol. 151, No. 2, 2003, pp. 330-337. doi:10.1007/s00221-003-1502-9

[36] F. Gandolfo, C. Li, B. J. Benda and C. P. Schioppa, E. Bizzi, "Cortical Correlates of Learning in Monkeys Adapting to a New Dynamical Environment," Proceedings of the National Academy of Sciences of the USA, Vol. 97, No. 5, 2000, pp. 2259-2263. doi:10.1073/pnas.040567097

[37] T. Hunter, P. Sacco, M. A. Nitsche and D. L. Turner, "Modulation of Internal Model Formation during Force Field-Induced Motor Learning by Anodal Transcranial Direct Current Stimulation of Primary Motor Cortex," The Journal of Physiology, Vol. 587, No. 12, 2009, pp. 2949-2961. doi:10.1113/jphysiol.2009.169284

[38] A. Hadipour-Niktarash, C. K. Lee, J. E. Desmond and R. Shadmehr, "Impairment of Retention but Not Acquisition of a Visuomotor Skill through Time-Dependent Disruption of Primary Motor Cortex," The Journal of Neuroscience, Vol. 27, No. 49, 2007, pp. 13413-13419. doi:10.1523/JNEUROSCI.2570-07.2007

[39] J. Xiao, C. Padoa-Schioppa and E. Bizzi, "Neuronal Correlates of Movement Dynamics in the Dorsal and Ventral Premotor Area in the Monkey," Experimental Brain Research, Vol. 168, No. 1-2, 2006, pp. 106-119. doi:10.1007/s00221-005-0074-2

[40] L. E. Sergio, C. Hamel-Paquet and J. F. Kalaska, "Motor Cortex Neural Correlates of Output Kinematics and Kinetics during Isometric-Force and Arm-Reaching Tasks," Journal of Neurophysiology, Vol. 94, No. 4, 2005, pp. 2353-2378. doi:10.1152/jn.00989.2004

[41] J. A. Rathelot and P. L. Strick, "Subdivisions of Primary Motor Cortex Based on Cortico-Motoneuronal Cells," Proceedings of the National Academy of Sciences of the USA, Vol. 106, No. 3, 2009, pp. 918-923. doi:10.1073/pnas.0808362106

[42] Y. Yanai, N. Adamit, Z. Israel, R. Harel and Y. Prut, "Coordinate Transformation Is First Completed Downstream of Primary Motor Cortex," The Journal of Neuroscien, Vol. 28, No. 7, 2008, pp. 1728-1732. doi:10.1523/JNEUROSCI.4662-07.2008 